\title{
Oléagineux et structures d'exploitations agricoles dans les PECO
}

Oléagineux, Corps Gras, Lipides. Volume 11, Numéro 4, 327-30, JUILLET-OCTOBRE 2004, CONSÉQUENCES DE LA RÉFORME

\author{
Auteur(s) : Emile Choné \\ AGROPOL (Association pour le développement international agronomique et industriel des \\ protéagineux et des oléagineux), 12, avenue George V 75008 Paris
}

Summary : The agriculture of the CEEC's is characterized by a strong diversity in the size of the farms. Oilseed crops are grown mainly by the big farms ( $80 \%$ in Poland) which take their origin in State farms. New big farms are under establishement by leasing of land from small owners (in Romania). The Common Agricultural Policy in the new member CEEC's will result in: little effect on restructuring of small farms, putting oilseeds aside from that sector, quick effect on the improvement of the productivity of oilseeds in the big farms and probably little influence on oilseed acreage expansion.

Keywords : CAP, CEEC's

\section{ARTICLE}

\section{Les PECO et leur agriculture}

Dix pays d'Europe centrale et orientale ont vu leur candidature agréée par l'Union européenne. Huit d'entre eux viennent d'entrer au $1^{\text {er }}$ mai 2004 (la Pologne, les trois Républiques baltes, la République tchèque, la Slovaquie, la Hongrie et la Slovénie). L'entrée de la Roumanie et de la Bulgarie est prévue en 2007.

À la suite de treize années de transition économique, les PECO accusent encore un retard économique par rapport à I'UE (tableau 1)( Tableau 1 ). Pour les deux plus grands pays, le PIB par habitant ne représente, en proportion de celui de l'UE à 15 , que $39,6 \%$ pour la Pologne et $24,6 \%$ pour la Roumanie.

L'agriculture manifeste également un retard de productivité par rapport au reste de l'économie. L'agriculture emploie 19,6\% des actifs en Pologne et ne représente que 3,1\% du PIB, 37,7\% des actifs en Roumanie pour $13 \%$ du PIB.

Le débat intense sur la restructuration nécessaire de l'agriculture des PECO ne doit pas occulter la diversité des exploitations agricoles de ces pays.

L'agriculture des PECO est en effet caractérisée par la coexistence d'une agriculture de microexploitations de subsistance et d'une agriculture sur de très grandes exploitations héritées du passé. 
Pour comprendre la situation présente, il est utile de revenir aux structures d'exploitation de la fin de la période communiste (Tableau 2( Tableau 2 )).

La collectivisation postérieure à la deuxième guerre mondiale avait été menée par la mise en place des coopératives agricoles au sein desquelles les paysans étaient généralement contraints d'apporter leurs terres et par la nationalisation pure et simple des grandes propriétés agricoles.

La seule exception notable à cette collectivisation fut la résistance des paysans polonais à apporter leurs terres aux coopératives.

Ces coopératives possédaient l'ensemble des moyens de production y compris la terre. On comprendra dès lors la répugnance profonde actuelle des agriculteurs des PECO au concept de coopérative et les explications nécessaires pour leur faire comprendre le succès des coopératives de services en Europe occidentale.

Partant de cette situation, la transformation des structures agricoles s'est faite selon des voies et à des vitesses différentes selon les pays à partir de 1990.

- En Pologne, la gestion des fermes d'Etat a été confiée dès le début de la période de transition à des privés, le transfert de la propriété des terres étant plus progressif et encore en cours. Ces grandes fermes d'Etat (les PGR, panstwowe gospodarstwa rolne) n'ont pratiquement pas été démantelées. Elles aboutissent aujourd'hui à de grandes ou très grandes exploitations agricoles privées.Les grandes régions qui furent sous régime allemand jusqu'à la deuxième guerre mondiale (Mazurie, Poméranie, Silésie) comptent une forte proportion de ces anciennes grandes fermes d'Etat.

- En Roumanie, la transition s'est passée en deux temps distincts. Au début de la transition économique a eu lieu la restitution des parts des coopératives aux ayants droit des terres correspondantes. Ce processus a conduit à attribuer à une large population des lopins de terre de 2 à 3 ha en moyenne. Beaucoup de ces nouveaux propriétaires résident en ville.En 2001, la situation était la suivante ( (figure 1) ): $68 \%$ de la surface agricole totale de Roumanie était constituée de petits propriétaires-exploitants à côté desquels subsistaient les fermes d'Etat sur $15 \%$ de la surface agricole totale (SAT). Sur $16 \%$ de la SAT, une partie des attributaires de lopins de terre avaient choisi de continuer à exploiter ensemble leurs terres au sein d'associations formelles ou familiales (dotées ou non d'une forme juridique).Ce n'est que récemment que les grandes fermes d'Etat ont été totalement privatisées (2002-2003). Les ayants droit des grandes propriétés nationalisées en 1947 n'ont récupéré qu'un maximum de 50 ha par propriétaire, le reste a été vendu à des privés.

Au cours des toutes dernières années s'amorce la constitution de grandes exploitations sous forme de sociétés privées qui louent les terres aux petits propriétaires privés. Il est courant de voir dans la plaine du Danube des sociétés d'exploitation agricole de un à plusieurs milliers d'hectares louant les terres à plusieurs centaines de propriétaires.

En tout état de cause, ces grandes exploitations désormais totalement gérées par des privés sont a priori bien placées pour diminuer leurs coûts sachant de plus qu'elles vont bénéficier progressivement des aides directes de la politique agricole commune attribuées dans les nouveaux Etats membres. Enfin, en Roumanie et en Bulgarie, elles bénéficient, d'ici l'adhésion de ces pays, des 
fonds SAPARD (Special Adhesion Programme for Agriculture and Rural Development) de l'Union européenne pour leur modernisation.

Tableau 1 PIB et agriculture des PECO en 2002

\begin{tabular}{|l|l|l|l|l|}
\hline & \multicolumn{2}{l|l}{ PIB par habitant } & \multicolumn{2}{l|}{ Agriculture } \\
\hline \hline en euros & en \% de l'UE15 & en \% du PIB & en \% des actifs & \\
\hline Bulgarie & 5900 & 24,6 & 12,5 & 10,7 \\
\hline République tchèque & 14400 & 60,0 & 3,7 & 4,9 \\
\hline Estonie & 10000 & 41,6 & 5,4 & 6,5 \\
\hline \hline Hongrie & 13600 & 56,6 & 4,3 & 6,0 \\
\hline \hline Lettonie & 8500 & 35,4 & 4,7 & 15,3 \\
\hline \hline Lituanie & 9400 & 39,2 & 7,1 & 18,6 \\
\hline Pologne & 9500 & 39,6 & 3,1 & 19,6 \\
\hline \hline Roumanie & 5900 & 24,6 & 13,0 & 37,7 \\
\hline \hline Slovaquie & 11400 & 47,5 & 4,5 & 6,6 \\
\hline \hline Slovénie & 17700 & 73,7 & 3,3 & 9,7 \\
\hline \hline UE15 & 24010 & 100 & & \\
\hline \hline UE25 & 21910 & & & \\
\hline \hline
\end{tabular}

Tableau 2 Structure des exploitations agricoles des PECO avant la transition économique

\begin{tabular}{|c|c|c|c|c|c|c|}
\hline & \multicolumn{2}{|l|}{ Coopératives } & \multicolumn{2}{|l|}{ Fermes d'Etat } & \multicolumn{2}{|c|}{$\begin{array}{l}\text { Exploitations individuelles } \\
\text { privées }\end{array}$} \\
\hline $\begin{array}{ll}\text { Part } & \text { SAT* }^{*} \\
(\%) & \end{array}$ & $\begin{array}{ll}\text { Taille moyenne } \\
\text { (ha) }\end{array}$ & \begin{tabular}{|ll||} 
Part & $\mathrm{SAT}^{\mathrm{a}}$ \\
$(\%)$ &
\end{tabular} & \begin{tabular}{|l} 
Taille moyenne \\
(ha)
\end{tabular} & \begin{tabular}{|ll} 
Part & $\mathrm{SAT}^{\mathrm{a}}$ \\
$(\%)$ &
\end{tabular} & Taille moyenne (ha) & \\
\hline Pologne & 4 & 335 & 19 & 3140 & 77 & 6,6 \\
\hline Hongrie & 80 & 4179 & 14 & 7138 & 6 & 0,3 \\
\hline $\begin{array}{l}\text { Rép. } \\
\text { tchèque }\end{array}$ & 61 & 2578 & 38 & 9443 & 0 & 5,0 \\
\hline
\end{tabular}




\begin{tabular}{|l||l||l|l|l|l|l|}
\hline $\begin{array}{l}\text { Rép. } \\
\text { slovaque }\end{array}$ & 69 & 2667 & 26 & 5186 & 5 & 0,3 \\
\hline Roumanie & 59 & 2374 & 29 & 5001 & 12 & 0,5 \\
\hline Bulgarie & 58 & 4000 & 29 & 1615 & 13 & 0,4 \\
\hline
\end{tabular}

${ }^{\mathrm{a} S u r f a c e}$ agricole totale.

\section{Les oléagineux et les structures d'exploitation}

Dans la plupart des PECO, la production d'oléagineux est majoritairement le fait de ces grandes exploitations agricoles, qu'elles soient issues des anciennes fermes d'Etat ou qu'elles fassent appel à la location de terres comme en Roumanie.

Cette caractéristique est liée :

- d'une part à la maîtrise ancienne des cultures oléagineuses, c'est le cas des régions polonaises de Mazurie, Poméranie et Silésie pour le colza ;

- d'autre part à la disponibilité des équipements spécifiques à ces cultures.

La répartition géographique du colza en Pologne ( (figure 2) ) est révélatrice des antécédents historiques et de la structure d'exploitation mentionnée plus haut. On estime dans ce pays que $80 \%$ du colza est produit par les anciens PGR.

Les oléagineux en Roumanie sont concentrés dans les zones les plus riches qui sont également celles des anciennes grandes fermes d'Etat et où se constituent de grandes exploitations agricoles privées en fermage ( (figure 3)).

\section{Évolution des exploitations de grandes cultures à l'occasion de l'élargissement}

Comme nous l'avons vu, les oléagineux des PECO sont produits principalement par les grandes exploitations.

Quel va être l'effet de la PAC sur la restructuration de l'agriculture des PECO en général et sur le développement de leurs oléagineux en particulier?

Le pays le plus concerné par la restructuration de son agriculture est la Pologne. Or ce pays vient de décider d'ouvrir les aides directes à toutes les exploitations agricoles de un hectare et plus. Dès lors, il est douteux que ces aides accélèrent la restructuration des petites exploitations. En conséquence, c'est toute une partie de la Pologne (centre, centre-sud et sud-est) qui ne sera pas concernée par le développement des oléagineux.

Pour les grandes exploitations céréalières de l'ensemble des PECO produisant la plus grande partie de oléagineux, avec l'accès aux aides directes de la PAC, au crédit bancaire pour optimiser les équipements et la conduite des cultures, et aux aides SAPARD, elles vont pouvoir accélérer leur modernisation et améliorer leur compétitivité en baissant leurs coûts de production. 
Dans bien des cas, elles disposent d'un avantage compétitif dû à leur taille comparée à celle des fermes de l'UE à 15.

Dans le processus de modernisation en cours, il est utile de mentionner le cas de la restauration des équipements d'irrigation en Roumanie qui vont améliorer la compétitivité de l'agriculture et permettre la relance de la production de soja. La Roumanie a en effet cultivé jusqu'à 500000 ha de soja à la fin de la période communiste. À cette époque, 3 millions d'ha de terres avaient été aménagés pour l'irrigation. On sait que $90 \%$ de ces aménagements ont été rendus inutilisables pour l'irrigation dès le début de la transition. La restauration de l'irrigation est en cours et devrait permettre à nouveau de produire du soja sur des surfaces potentiellement comparables à celles ayant existé dans le passé.

\section{Le rôle de l'organisation professionnelle dans l'évolution des oléagineux}

Tous les PECO disposent d'une filière oléagineuse avec en aval de la production des usines de trituration et de raffinage des huiles. L'organisation de la filière sous la période communiste avait privilégié le transfert direct des graines des grandes exploitations aux huileries sans prise en charge financière par un organisme collecteur intermédiaire, le stockage étant assuré, à façon, pour le compte du triturateur.

L'absence d'organisme collecteur acheteur se fait sentir actuellement, les triturateurs devant assurer dès la moisson l'achat de toute la récolte et son stockage pour toute la campagne.

Autre conséquence de cette organisation : le triturateur passe un grand nombre de contrats directement avec les producteurs agricoles avec les inconvénients d'une dissymétrie de la concentration de la demande par rapport à celle de l'offre et une différence d'accès à l'information et aux outils de marché.

Ceci amène les producteurs à s'organiser. Les producteurs de colza polonais ont mis en place avec I'appui d'Agropol le KZPR (Krajowe Zrzeszenie Producentow Rzepaku: Association nationale des producteurs de colza) afin de pallier ces inconvénients. En République tchèque, l'organisation a été poussée plus loin par le regroupement de tous les opérateurs de toute la filière avec le SPZO (Svaz Pěstitelů a Zpracovatelů Olejnin : Union des producteurs et transformateurs d'oléagineux) qui gère des actions d'intérêt commun dont celle de l'appui technique aux producteurs d'oléagineux.

\section{L'élargissement et l'équilibre des productions}

L'arrivée des PECO, encore très marqués par leur activité agricole, et à la recherche de tous les leviers de développement possibles, justifie de reposer la question de l'équilibre du développement des grandes cultures tant en termes d'adéquation au marché qu'en termes d'équilibre des productions et des assolements.

La reconquête du marché de I'UE des MRP et des huiles est un cas d'école. Faut-il continuer à ouvrir ce marché au tout soja importé et à cantonner ces pays à la production de céréales dont l'excédent, à l'échelle de l'UE, devra être exporté ? 


\section{Conclusion}

Au total, l'élargissement et la politique agricole commune vont avoir un impact sur les structures d'exploitations agricoles surtout par le renforcement des exploitations agricoles les plus importantes. On peut en attendre une amélioration sensible de la productivité des oléagineux mais une extension limitée des surfaces. Le marché du biodiesel en pleine expansion dans l'UE par son effet sur les prix peut déplacer vers les oléagineux l'équilibre des grandes cultures.

En tout état de cause, l'application de la PAC dans les PECO ne changera pas significativement le déficit en MRP de l'UE.

\section{Illustrations}

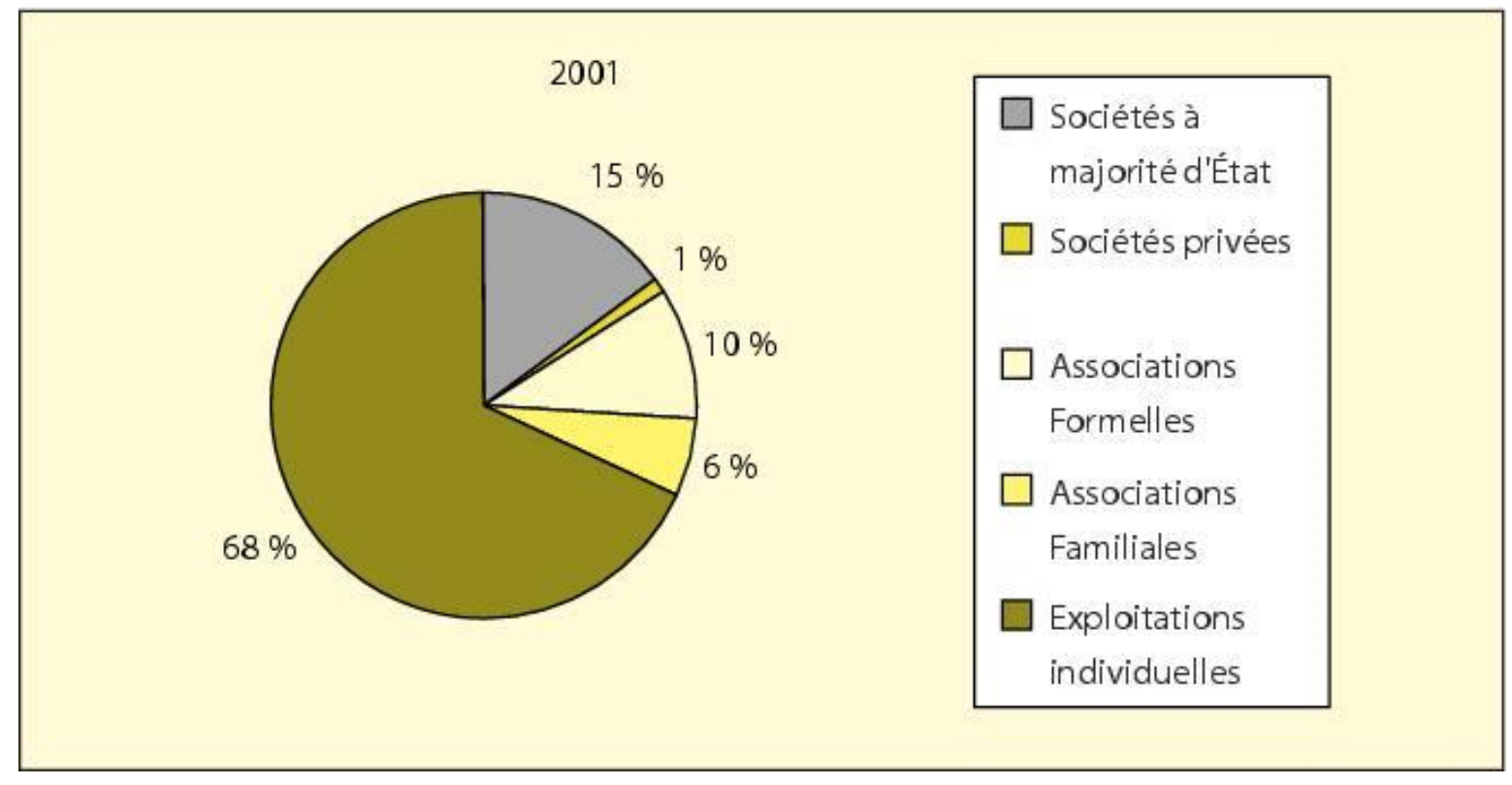

Figure 1 Evolution de la structure des exploitations agricoles en Roumanie. 


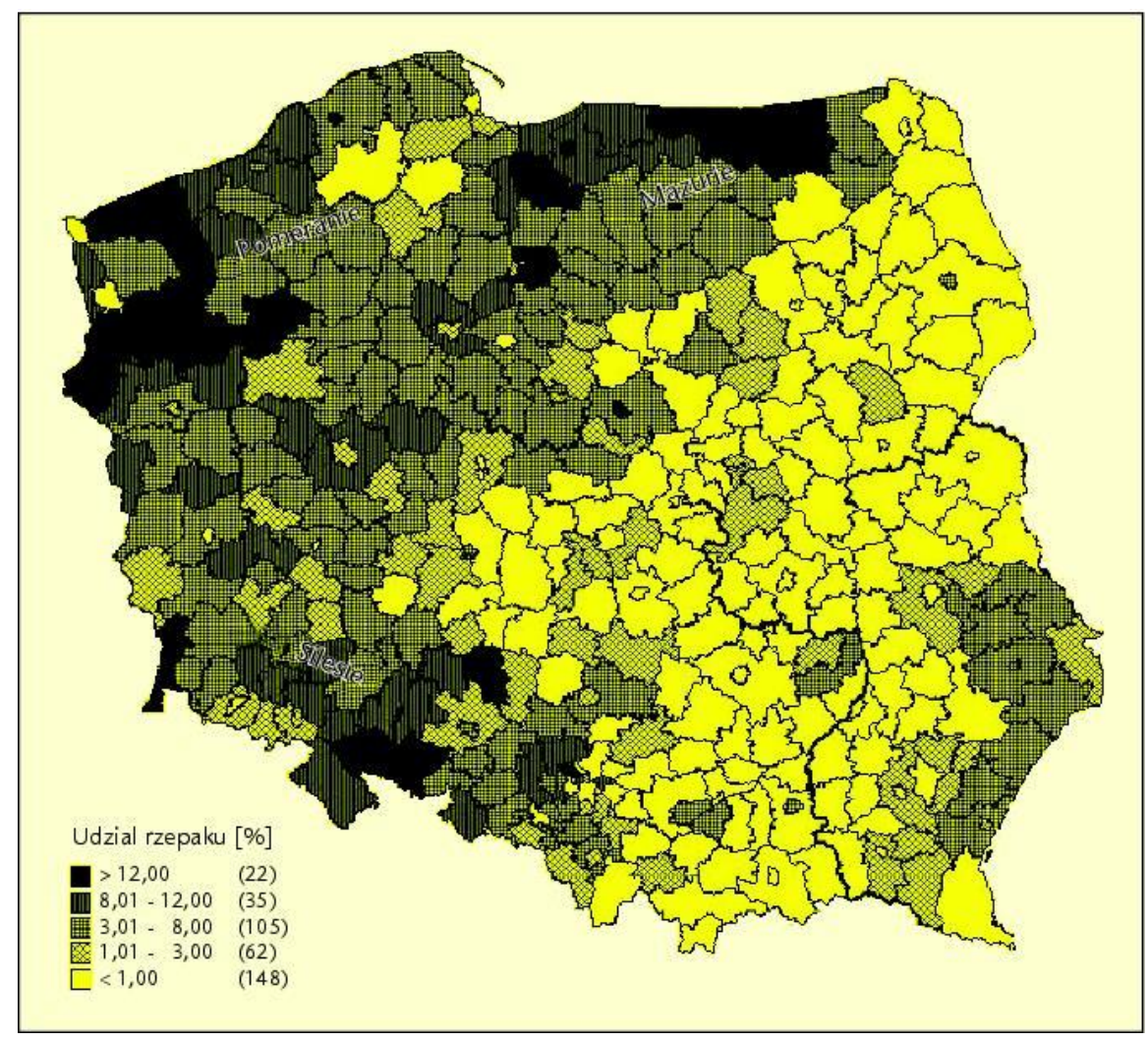

Figure 2 Pologne - Proportion du colza dans les surfaces semées en 2002 par Powiat.Carte dressée par lung Pulawy.

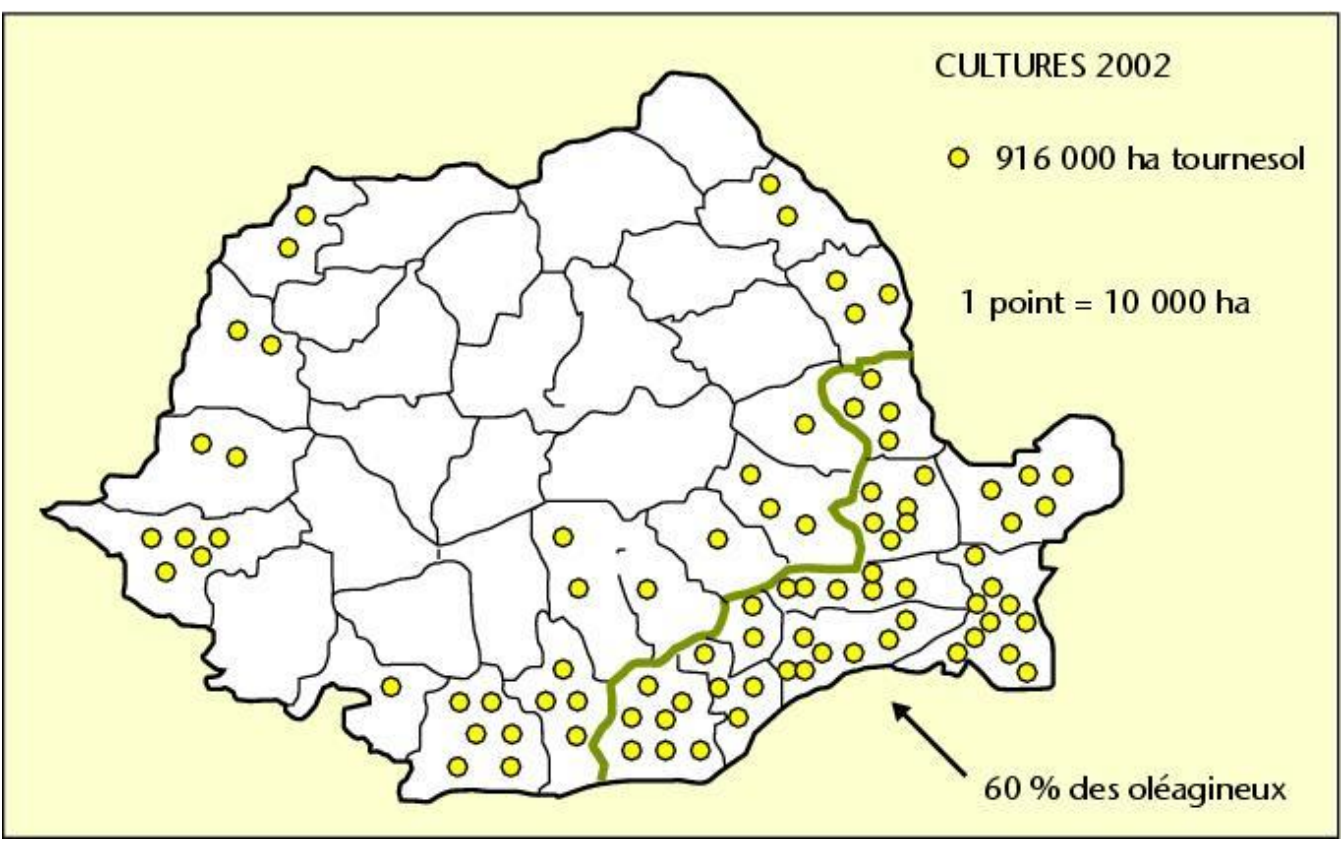

Figure 3 Roumanie - Répartition du tournesol en 2002. 
